\section{耍 Heighten Science P U B L I C I T I O N S Corporation ISSN 2576-9529}

\title{
Chronic kidney disease in women: a cross sectional screening in a tertiary care hospital in Varanasi
}

\author{
Rai Pradeep K*, Rai Punam² and Bedi Sonam ${ }^{3}$ \\ 'Senior Consultant Nephrologist, Department of Nephrology, Opal Hospital, Kakarmatta, \\ Varanasi- 221004, India \\ ${ }^{2}$ Senior Clinical Physiologist \& Infertility Expert, Opal Hospital, Kakarmatta, Varanasi- 221004, \\ India \\ ${ }^{3}$ Senior Data Analyst, Department of Medicine, Institute of Medical Sciences, Banaras Hindu \\ University, Varanasi- 221005, India
}

\begin{abstract}
*Address for Correspondence: Dr. Pradeep Kumar Rai, MBBS, MD, DM, D.N.B, F.A.S.N, Senior Consultant Nephrologist, Department of Nephrology, Opal Hospital, Kakarmatta, Varanasi, 221004, Uttar Pradesh, India, Tel: +91 9336913486; Email: pradnephro@gmail.com; pradeepk.rai@gmail.com
\end{abstract}

Submitted: 20 December 2018

Approved: 17 January 2019

Published: 18 January 2019

Copyright: @ 2019 Rai Pradeep K, et al. This is an open access article distributed under the Creative Commons Attribution License, which permits unrestricted use, distribution, and reproduction in any medium, provided the original work is properly cited

Keywords: Albuminuria; Chronic kidney disease; Diabetes mellitus; Glomerular filtration rate

\section{Abstract}

Background: Chronic Kidney Disease is no longer considered just a health burden today but a major health priority owing to its high treatment costs and poor outcome. World Kidney Day and International Women's Day in 2018 coincided, thus offering an opportunity to reflect on the importance of women's health, and specifically their kidney health. The current study aims to identify chronic kidney disease in women through a cross sectional screening in hospital based camp in Varanasi on World Kidney Day.

Methods: 138 females attending a health camp were screened for clinical parameters like serum creatinine, random blood sugar, and pregnancy complications. Demographic and anthropometric parameters were noted. Ordinal logistic regression analysis was used to find the predictors of chronic kidney disease stages.

Results: The median age of the participants was 36 years. The prevalence of chronic kidney disease was $6.4 \%$ with $1.4 \%$ of Stage $1,2.1 \%$ of Stage $2,1.4 \%$ of Stage 3 and $1.4 \%$ of Stage 4 . Women with diabetes were nearly 4 times at a greater risk of developing higher stages of chronic kidney disease compared to women without it. With a unit increase in serum creatinine levels, women were nearly 13 times more likely to develop higher stages of chronic kidney disease.

Conclusion: Serum creatinine levels and diabetes were the significant predictors for the development of higher stages of chronic kidney disease. Early screening for kidney diseases in women could reduce the load of health care delivery system that is involved in renal replacement therapies.

\section{Introduction}

Chronic Kidney Disease (CKD) is considered as a major health priority owing to its high treatment costs and poor outcome. It affects $\sim 10 \%$ of the world's adult population [1]. Even the early stages of CKD are known to be substantially associated with increased risks of cardiovascular morbidity, and premature mortality and decreased quality of life. Due to its asymptomatic nature at early stages, CKD is generally detected at advanced stages, resulting in the loss of opportunities to influence its future course of outcome. World Kidney Day and International Women's Day in 2018 coincided, thus offering an opportunity to reflect on the importance of women's health, and specifically their kidney health, to the community and the next generations, as well as to strive to be more curious about the unique aspects of kidney disease in women, so that we may apply those learning more broadly [1]. Females contribute to half of the society thus playing major role in the community health. 
Women have unique risks for kidney diseases [1], as men may have higher level of kidney function at the same level of serum creatinine. Even less attention is given to sex differences in nephrology research, despite evidence of sex-specific differences in kidney function and in the epidemiology, diagnosis, underlying mechanisms, natural history and outcomes of CKD, as well as the need to consider the reproductive health of affected women and the renal and wider health of their offspring [2].

In the year 2002, the Kidney Disease Outcomes Quality Initiative (KDOQI) of the National Kidney Foundation (NKF) developed a practice guideline for CKD, recommending routine testing for detection of kidney disease during general health check-ups for individuals at risk [3,4].

Progression of CKD and other adverse outcomes can be prevented or delayed through early detection and treatment modalities [5]. The estimated age-adjusted incidence rate of end stage renal disease (ESRD) is 229 per million population (pmp) whereas more than 1 lakh new patients enter renal replacement programs annually in India [6]. But, because of scarce resources, only 10\% of the these ESRD patients receive any form of renal replacement therapy (RRT) $[7,8]$. The lack of communitybased screening programs for CKD, results in patient detection at advanced stages [911]. In various locations around the world, access to education and medical care is not equitable amongst men and women; women remain underrepresented in many clinical research studies, thus limiting the evidence based on which to make recommendations to ensure best outcomes [1]. Hence, this study aims to identify chronic kidney disease in women through a cross-sectional screening in a hospital-based camp at a tertiary care center in Varanasi on World Kidney Day (March 8, 2018).

\section{Material and Methods}

\section{Data characteristics}

The study was conducted in the department of Nephrology at Opal hospital, Varanasi on occasion of the World Kidney day and International women's day. Considering 15\% prevalence of CKD in females [12-14], the sample size was estimated to be 138 females. A total of 141 women were considered in the study sample. Consent was obtained from each study participant. Opal Hospital Ethics Committee approval was taken before starting the present study. Subjects symptomatic for acute kidney injury (AKI) were excluded from the study. Demographic (i.e. age), and clinical characteristics, history of any other co morbidities, pregnancy complications and details, history of diabetes mellitus (DM) and hypertension (HTN) were noted. Anthropometric parameters i.e. height, weight and waist-hip ratio (WHR) were recorded by the standard methods. A random venous blood sample was drawn and was analyzed for serum creatinine, random blood sugar (RBS) and uric acid. Finally urine was tested for the presence of albuminuria for each subject.

\section{Definitions of variables}

Some of the variables were converted into categories for appropriate clinical interpretation. Age was divided into two categories, e.g. $<36$ and $\geq 36$ years based on median values. Individuals with body mass index (BMI) up to 18.49 were considered as underweight, $18.50-24.99$ as normal, $25.00-29.99$ overweight and $\geq 30.00\left(\mathrm{~kg} / \mathrm{m}^{2}\right)$ as obese.

DM was defined as the use of glucose lowering medicine, or RBS $\geq 200 \mathrm{mg} / \mathrm{dl}$ along with presence of osmotic symptoms. Systolic and diastolic blood pressures (BP) were measured in a standard way. HTN was defined as the use of antihypertensive medication, or systolic BP $\geq 140 \mathrm{mmHg}$, or diastolic BP $\geq 90 \mathrm{mmHg}$. Abdominal obesity was defined as a waist hip ratio above 0.85 as per the World Health Organisation norms. Information on number of children surviving was taken on scale variable and further dichotomized as: $(<3$ and or $\geq 3$ ). Delivery was categorized as vaginal or caesarean and delivery place as home or hospital. 
Serum creatinine was recorded as scale variable and further subdivided in 2 groups: $<1.30$ and $\geq 1.30(\mathrm{mg} / \mathrm{dl})$ for checking association with the outcome variable. A urine dipstick (Medi-Test Combi 9-Macherey Nagel, Duren, Germany) was performed for each participant. Uric acid level was considered normal between $2.4-6.0 \mathrm{mg} / \mathrm{dL}$. It was categorized as normal and abnormal. According to the KDIGO (Kidney Disease: Improving Global Outcomes) guidelines [15], the CKD outcome was identified based on estimated glomerular filtration rate (eGFR) and albuminuria. Albuminuria was regarded as significant if $1+$ and above. GFR was calculated using the Modification of Diet in Renal Disease (MDRD) study equation. CKD stages were defined as: stage 1 if eGFR $\geq 90$ and albuminuria present; stage 2 if eGFR $=60$ to 89 and albuminuria present; stage 3 if eGFR= 30 to 59 ; stage 4 if eGFR $=15$ to 29 ; and stage 5 if eGFR $<15$. All individuals with a positive test result for chronic kidney dysfunction in the screening were referred to the nephrologists without any further follow up.

\section{Statistical analysis}

All the statistical analysis was performed on Stata/IC V10.1 (StataCorp., College Station, TX, USA). In the descriptive analyses, means \pm SDs, medians (inter-quartile range [IQR]) were calculated for continuous data, and proportions for categorical data. The association of each variable with the disease outcome was seen separately using Pearson's Chi square test and strength of association using Cramer V statistics. Significant mean differences in the continuous variables were also checked using Kruskal Wallis test before categorizing them.

A two-step procedure was used to select the most appropriate predictive model for the occurrence of different CKD stages. Since the study outcome (CKD) was divided into 4 categories (1-4, each depicting stages of CKD) according to the severity of disease hence an ordinal regression analysis was performed. Initially bi-variable ordinal regression analysis was run and finally multi-variable ordinal regression including only those significant at $\mathrm{p}=0.10 \mathrm{in}$ bi-variable analysis. Stepwise backward likelihood ratio regression was performed to eliminate insignificant predictors of CKD in multi-variable regression and p-value of 0.05 was used as a threshold for predictors to contribute significantly. 95\% confidence intervals of the estimated odds ratio (OR) was also obtained along with the corresponding $\mathrm{p}$-values.

\section{Results}

The demographics, clinical and laboratory data are summarized in table 1 . The median (IQR) age of the participants was 36 (28-44) years. Nearly half (47.5\%) of the subjects were younger than 36 years. About one-third (28.6\%) reported individual or familial history of renal diseases or any other co-morbidities. Based on BMI, 7.1\%, $35.5 \%$ and $14.9 \%$ females were underweight, overweight and obese respectively while $42.6 \%$ had a normal BMI.

HTN were prevalent in approximately one fifth $(19.1 \%, \mathrm{n}=27)$ of the cohort and was statistically significant with the outcome $(\mathrm{p}=0.029) .7 .4 \%$ hypertensive subjects were suffering with Stage 4 CKD. Diabetes mellitus was found in only 2 subjects (1.5\%) and both lying in CKD stages 1 and 2 ( $\mathrm{p}<0.0001$ ). 98.5\% females had abdominal obesity. Pregnancy complications were reported by $20(14.9 \%)$ females. Menstrual problems were found in only $9(6.7 \%)$ females. Nearly one-third $(31.7 \%, \mathrm{n}=40)$ females had at least one home delivery without any trained personnel. None of the study participants reported to have undergone cervix cancer vaccination. $15.4 \%$ females reported to have had at least one caesarean delivery. The study subjects reported a median number of children as 2 (IQR= $2-3$ ) although $44.2 \%$ had multiple pregnancies with $\geq 3$ surviving children.

The prevalence of CKD was 6.4\% (95\% CI: $2.3-10.5 \%$ ) in the whole cohort with $1.4 \%$ of Stage 1, 2.1\% of Stage 2, $1.4 \%$ of Stage 3 and $1.4 \%$ of Stage 4 (Table 2). There were no subjects with Stage 5 CKD. The serum creatinine levels of only 2 
Table 1: Profile of female subjects according to chronic kidney disease outcome.

\begin{tabular}{|c|c|c|c|c|c|c|}
\hline \multirow{2}{*}{ Characteristics } & \multirow{2}{*}{ N (\%) } & \multicolumn{4}{|c|}{$\%$ CKD staging } & \multirow{2}{*}{$\begin{array}{c}\text { Pearson's } x^{2} \\
\text { p-value }\end{array}$} \\
\hline & & 1 & 2 & 3 & 4 & \\
\hline \multicolumn{7}{|l|}{ Age (years) } \\
\hline$<36$ & $67(47.5)$ & 0.0 & 1.5 & 0.0 & 0.0 & \multirow{2}{*}{0.199} \\
\hline$\geq 36$ & $74(52.5)$ & 2.7 & 2.7 & 2.7 & 2.7 & \\
\hline \multicolumn{7}{|l|}{ BMI } \\
\hline Underweight & $10(7.1)$ & 0.0 & 0.0 & 0.0 & 10.0 & \multirow{4}{*}{0.221} \\
\hline Normal & $60(42.6)$ & 0.0 & 1.7 & 0.0 & 1.7 & \\
\hline Overweight & $50(35.5)$ & 4.0 & 4.0 & 4.0 & 0.0 & \\
\hline Obese & $21(14.9)$ & 0.0 & 0.0 & 0.0 & 0.0 & \\
\hline \multicolumn{7}{|l|}{ Abdominal obesity } \\
\hline Yes & $132(98.5)$ & 1.5 & 2.3 & 1.5 & 1.5 & \multirow{2}{*}{0.997} \\
\hline No & $2(1.5)$ & 0.0 & 0.0 & 0.0 & 0.0 & \\
\hline \multicolumn{7}{|l|}{ DM } \\
\hline Yes & $2(1.5)$ & 50.0 & 50.0 & 0.0 & 0.0 & $<0.001$ \\
\hline No & $135(98.5)$ & 0.7 & 1.5 & 1.5 & 1.5 & \\
\hline \multicolumn{7}{|l|}{ HTN } \\
\hline Yes & $27(19.1)$ & 3.7 & 3.7 & 0.0 & 7.4 & \multirow{2}{*}{0.029} \\
\hline No & $114(80.9)$ & 0.9 & 1.8 & 1.8 & 0.0 & \\
\hline \multicolumn{7}{|l|}{ Uric acid } \\
\hline Normal & $133(95.0)$ & 1.5 & 2.3 & 1.5 & 1.5 & \multirow{2}{*}{0.973} \\
\hline Abnormal & $7(5.0)$ & 0.0 & 0.0 & 0.0 & 0.0 & \\
\hline \multicolumn{7}{|c|}{ History of co morbidities } \\
\hline Yes & $40(28.6)$ & 2.5 & 5.0 & 2.5 & 0.0 & \multirow{2}{*}{0.418} \\
\hline No & $100(71.4)$ & 1.0 & 1.0 & 1.0 & 2.0 & \\
\hline \multicolumn{7}{|c|}{ History of pregnancy complications } \\
\hline Yes & $20(14.9)$ & 0.0 & 5.0 & 0.0 & 0.0 & \multirow{2}{*}{0.761} \\
\hline No & $114(85.1)$ & 1.8 & 1.8 & 1.8 & 1.8 & \\
\hline \multicolumn{7}{|l|}{ Menstrual problems } \\
\hline Yes & $9(6.7)$ & 0.0 & 0.0 & 0.0 & 0.0 & 0952 \\
\hline No & $125(93.3)$ & 1.6 & 2.4 & 1.6 & 1.6 & \\
\hline Operation type & & & & & & \\
\hline Normal & $104(84.6)$ & 1.9 & 1.9 & 1.9 & 1.9 & \\
\hline Caesarean & $19(15.4)$ & 0.0 & 0.0 & 0.0 & 0.0 & 0.815 \\
\hline Delivery place & & & & & & \\
\hline Home & $40(31.7)$ & 2.5 & 2.5 & 5.0 & 2.5 & \\
\hline Hospital & $86(68.3)$ & 1.2 & 2.3 & 0.0 & 1.2 & 0.277 \\
\hline Children no. & & & & & & \\
\hline$<3$ & $72(55.8)$ & 0.0 & 2.8 & 1.4 & 0.0 & \\
\hline$\geq 3$ & $57(44.2)$ & 3.5 & 1.8 & 1.8 & 3.5 & 0.252 \\
\hline Serum creatinine & & & & & & \\
\hline$<1.30$ & $139(98.6)$ & 1.4 & 2.2 & 1.4 & 0.0 & \\
\hline$\geq 1.30$ & $2(1.4)$ & 0.0 & 0.0 & 0.0 & 100.0 & $<0.001$ \\
\hline Glomeruler filtratio & & & & & & \\
\hline$\geq 90$ & $101(71.6)$ & 2.0 & 0.0 & 0.0 & 0.0 & \\
\hline $60-89$ & $36(25.5)$ & 0.0 & 8.3 & 0.0 & 0.0 & \\
\hline $30-59$ & $2(1.4)$ & 0.0 & 0.0 & 100.0 & 0.0 & $<0.001$ \\
\hline$<30$ & $2(1.4)$ & 0.0 & 0.0 & 0.0 & 100.0 & \\
\hline Albuminuria & & & & & & \\
\hline Yes & $7(5.0)$ & 28.6 & 42.9 & 0.0 & 28.6 & \\
\hline No & $134(95.0)$ & 0.0 & 0.0 & 1.5 & 0.0 & $<0.001$ \\
\hline
\end{tabular}

Table 2: The chronic kidney disease staging outcome.

\begin{tabular}{|c|c|}
\hline CKD event & $\mathbf{N}(\%)$ \\
\hline Absent & $132(93.6 \%)$ \\
\hline Stage 1 & $2(1.4 \%)$ \\
\hline Stage 2 & $3(2.1 \%)$ \\
\hline Stage 3 & $2(1.4 \%)$ \\
\hline Stage 4 & $2(1.4 \%)$ \\
\hline
\end{tabular}


(1.4\%) participants were $\geq 1.30 \mathrm{mg} / \mathrm{dl}$ both from Stage 4 CKD and hence significantly associated with CKD $(\mathrm{p}<0.0001)$ as well. Uric acid was found to be elevated in $5 \%$ subjects. $71.6 \%$ participants were falling in eGFR $\geq 90,25.5 \%$ in eGFR between $60-89$ and $1.4 \%$ each in eGFR between 30-59 and below 30. eGFR was highly associated with CKD ( $\mathrm{p}<0.0001)$. Albuminuria was found positive in only $5 \%(\mathrm{n}=7)$ individuals and statistically associated with CKD occurrence $(\mathrm{p}<0.0001)$. Cramer V statistics indicated, the strength of association of serum creatinine, eGFR, albuminuria, DM and HTN with the study outcome (CKD stages) was highly significant $(\mathrm{p}<0.05)$ and to the extent of $100 \%, 83 \%, 100 \%, 63.9 \%$ and $27.7 \%$ respectively. Albuminuria and eGFR were highly associated with CKD $(\mathrm{p}<0.001)$ but being prelude for CKD occurrence, were not considered in the regression model.

Predictors like age, BMI, and history of co morbidities, DM, HTN, uric acid, serum creatinine levels, menstrual problems, pregnancy complications, delivery place, and type of operation were considered for the regression model. In the bi-variable ordinal regression analysis BMI, creatinine, DM (at 5\%) and age, numbers of children, HTN (at $10 \%$ ) were independent predictors for CKD. Subsequently, these were considered for multi-variable ordinal regression analysis.

Women with diabetes had nearly 4 times greater risk of developing higher stages of CKD (Table 3: OR= 4.204, 95\% CI=1.058-7.350) compared to women without it. Diabetes was also associated with albuminuria $(p=0.002)$ and history of co morbidities $(\mathrm{p}=0.081)$.

With a unit increase in serum creatinine levels, women were 13 times more likely to develop higher stages of CKD (Table 3: OR=13.569, 95\% CI= 5.503-21.636). Hypertension $(\mathrm{p}=0.036)$, albuminuria $(\mathrm{p}=0.002)$, eGFR categories $(\mathrm{p}<0.001)$ were significantly associated with higher levels of creatinine $(\geq 1.30 \mathrm{mg} / \mathrm{dl})$.

\section{Discussion}

Delayed recognition and treatment of CKD may predispose patients to adverse future outcomes. The objective of the present study was to describe the prevalence patterns and association with some known risk factors of CKD among women at screening levels in a hospital based screening camp.

Only 2 subjects reported a high serum creatinine level $(\geq 1.30 \mathrm{mg} / \mathrm{dl})$ and both of these were hypertensive $(p=0.036)$. Similarly, both these females were having significant albuminuria and with eGFR $<30$, hence there was clear cut implication of the CKD outcome $(p<0.001)$. Both the females had undergone multiple pregnancies with $\geq 3$ children surviving although it was not significantly associated $(p=0.252)$ with the outcome. Serum creatinine levels were significantly different across the 4 stages of CKD (One way ANOVA $\mathrm{p}<0.001$ ). Ordinal logistic regression analysis depicted similar findings. Unit increase in serum creatinine levels increases the chance of falling in higher stages of CKD by more than 13 times (Table 3: $\mathrm{OR}=13.569, \mathrm{CI}=5.503-21.636$ ).

Only 2 subjects were suffering with DM (1.4\%). Both women with diabetes were $\geq 36$ years of age, overweight, having abdominal obesity, history of co morbidities, and proteinuria. Although the association was significant only with proteinuria $(p=0.002)$. Ordinal regression demonstrated nearly 4 times higher risk in women with diabetes to lie in the higher stages of CKD (Table 3: OR=4.204, CI=1.058-7.350). Women with diabetes also had other co-morbid conditions $(\mathrm{p}=0.081)$, which could partly explain

Table 3: Predictors of different levels of chronic kidney disease: A Multi-variable ordinal regression analysis.

\begin{tabular}{|c|c|c|c|c|c|}
\hline Characteristics & OR & SE & Wald & P value & $95 \%$ Cl of OR \\
\hline Serum creatinine & 13.569 & 4.116 & 10.871 & 0.001 & $5.503-21.636$ \\
\hline DM (Ref: No) & 4.204 & 1.605 & 6.859 & 0.009 & $1.058-7.350$ \\
\hline Yes &
\end{tabular}


the high prevalence of higher stages of CKD in this group. In western countries, DM account for over $2 / 3^{\text {rd }}$ of the cases of CKD. In India too, DM accounts for $40-60 \%$ cases of CKD [16]. India is observing fast demographic transition, moreover, the life style e.g. food habits are changing unfavorably and physical activity is reducing. Thus, the situation of asymptomatic CKD subjects along with DM is growing up. Under such conditions the findings of the present study, though, cannot be generalized but gives the signal to screen female population (both young and elderly) at mass level for CKD detection to avoid the adverse outcomes in future lives.

Diabetes was a significant predictor for higher CKD stages in females. Similar literature has been reported earlier [6,13,17]. Diabetes may insist alterations in sex hormone levels. Studies have reported that diabetes can lead to an imbalance in sex hormone levels; however, whether these changes correlate with the decline in renal function associated with diabetes is unclear. Furthermore, diabetic renal disease rarely develops before puberty, and the onset of puberty accelerates microalbuminuria, supporting the idea of the involvement of sex hormones in the development and progression of the disease [18].

The equality of access to medicare facilities to women is of concern especially in low and middle income countries because, they are disadvantaged by discrimination rooted in socio-cultural factors [1,2]. There is a paucity of information about sex differences in CKD treatment, but in many countries, men are reported to be more likely than women to receive any form of RRTs [19].

In the present study all the CKD cases were detected with multiple pregnancies ( $p=0.034$ ), biologically the presence of any degree of CKD has a negative effect on pregnancy, and given the increase in risk of CKD progression postpartum, it raises challenging ethical issues around conception and maintenance of pregnancies. Various potential adverse effects of pregnancy on kidney health have been witnessed in prior studies [1].

We hope this paper will increase awareness of the considerations and challenges specific to the renal health of women, as well as encourage further research into this important area.

\section{Acknowledgement}

We would like to thank medical officers and staff of Opal hospital: Dr Shashi Kumar Gupta, Dr Hemant Kumar Rai, Dr Zafar Raja, Dr Surendra Nath Gupta, Mrs. Arti Maurya and Mr. Bansi Dhar Pandey who have helped with evaluation of subjects in the screening camp conducted at Opal hospital.

\section{Declarations}

- $\quad$ Ethics approval and consent to participate

The subjects who consented to participate in the study were considered.

Opal Hospital Ethics Committee approval was taken before starting the present study.

- $\quad$ Competing interests

The authors declare that they have no competing interests

- $\quad$ Funding

Not applicable

\section{References}

1. Piccoli GB, Alrukhaimi M, Liu ZH, Zakharova E, Levin A, et al. Women and kidney disease: reflections on World Kidney Day 2018. Kidney Int. 2018; 93: 278-283. Ref.: https://goo.gl/J8WNB9 
2. Luyckx VA, Brenner BM. Birth weight, malnutrition and kidney-associated outcomes-a global concern. Nat Rev Nephrol. 2015; 11: 135-139. Ref.: https://goo.gl/Rezeuq

3. Levey AS, Coresh J, Balk E, Kausz AT, Levin A, et al. National Kidney Foundation practice guidelines for chronic kidney disease: evaluation, classification, and stratification. Ann Intern Med. 2003; 139: 137-147. Ref.: https://goo.gl/t2V8cj

4. Woodhouse S, Batten $\mathrm{W}$, Hendrick H, Malek PA. The glomerular filtration rate: an important test for diagnosis, staging, and treatment of chronic kidney disease. Lab Med. 2015; 37: 244-246. Ref.: https://goo.gl/XeVH4U

5. Ležaić V, Bajčetić S, Peruničić-Peković G, Bukvić D, Dimković N, et al. Screening of elderly for chronic kidney disease. Kidney Blood Press. Res. 2012; 35: 497-503. Ref.: https://goo.gl/oPwqUH

6. Singh AK, Farag YM, Mittal BV, Subramanian KK, Reddy SR, et al. Epidemiology and risk factors of chronic kidney disease in India-results from the SEEK (Screening and Early Evaluation of Kidney Disease) study. BMC Nephrol. 2013; 14: 114. Ref.: https://goo.gl/kcqhkG

7. Ruggenenti P, Schieppati A, Remuzzi G. Progression, remission, regression of chronic renal diseases Lancet. 2001; 357: 1601-1608. Ref.: https://goo.gl/WFfKdR

8. Kher V. End-stage renal disease in developing countries. Kidney Int. 2002; 62: 350-362. Ref.: https://goo.gl/4tbJ2u

9. Jha V. End-stage renal care in developing countries: the India experience. Ren Fail. 2004; 26: 201208. Ref.: https://goo.gl/wucW1c

10. Modi GK, Jha V. The incidence of end-stage renal disease in India: a population-based study. Kidney Int. 2006; 70: 2131-2133. Ref.: https://goo.gl/FQ1RQm

11. Sakhuja V, Sud K. End-stage renal disease in India and Pakistan: burden of disease and management issues. Kidney Int. 2003; 63: S115-S118. Ref.: https://goo.gl/9NXFyY

12. Saran R, Robinson B, Abbott KC, Agodoa LY, Albertus P. et al. US Renal Data System 2016 annua data report: epidemiology of kidney disease in the United States. Am J kidney Dis. 2017; 69: A7-A8. Ref.: https://goo.gl/awrkmj

13. Stats F. National Chronic Kidney Disease Fact Sheet. 2017; Ref.: https://goo.gl/uKezR9

14. Pounds LL, Teodorescu VJ. Chronic kidney disease and dialysis access in women. J Vasc Surg. 2013; 57: 49S-53S. Ref.: https://goo.gl/Hmn4aS

15. Levey AS, Eckardt KU, Tsukamoto Y, Levin A, Coresh J, et al. Definition and classification of chronic kidney disease: a position statement from Kidney Disease: Improving Global Outcomes (KDIGO). Kidney Int. 2005; 67: 2089-2100. Ref.: https://goo.gl/BmRZTL

16. Varma PP. Prevalence of chronic kidney disease in India-Where are we heading? Indian J Nephrol. 2015; 25: 133-135. Ref.: https://goo.gl/GQNFZX

17. Webster AC, Nagler EV, Morton RL, Masson P. Chronic kidney disease. Lancet. 2017; 389: 12381252. Ref.: https://goo.gl/bCDzNv

18. Maric C. Sex, diabetes and the kidney. Am J Physiol Physiol. 2009; 296: F680-F688. Ref.: https://goo.gl/x962ZS

19. Carrero JJ, Hecking M, Chesnaye NC, Jager KJ. Sex and gender disparities in the epidemiology and outcomes of chronic kidney disease. Nat Rev Nephrol. 2018; 14: 151-164. Ref.: https://goo.gl/jWwF6D 